\title{
Sistema de innovación como marco analítico de la agricultura protegida en la región centro de México*
}

Cómo citar este artículo : García-Sánchez, E. I., Vargas-Canales, J. M., Palacios-Rangel, M. I., y Aguilar-Ávila, J. (20r8). Sistema de innovación como marco analítico de la agricultura protegida en la región centro de México. Cuadernos de Desarrollo Rural, I5 (8I), I-24. https://doi.org/I0.III44/Javeriana.cdri5-8I.sima

Edgar Iván García-Sánchez

Centro de Investigaciones Interdisciplinarias sobre

Desarrollo Regional, México

ORCID: http://orcid.org/0000-000I-5774-4I99

Juan Manuel Vargas-Canales

Universidad de Guanajuato, México

jm.vargas@ugto.mx

ORCID: http://orcid.org/0000-0003-I918-9395

María Isabel Palacios-Rangel

Centro de Investigaciones Económicas, Sociales y

Tecnológicas de la Agroindustria y la Agricultura

Mundial, México

ORCID: http://orcid.org/0000-000I-9382-863X

Jorge Aguilar-Ávila

Centro de Investigaciones Económicas, Sociales y

Tecnológicas de la Agroindustria y la Agricultura

Mundial, México

ORCID: http://orcid.org/0000-0002-6129-7050

DOI: https://doi.org/I0.III44/Javeriana.cdri5-8I.sima

Redalyc: http://www.redalyc.org/articulo.oa?id=II757604006

Recibido: 03 de Noviembre de 2017 I Publicación: I8 de junio de 2018

\section{Resumen:}

Esta investigación tuvo como objetivo analizar la agricultura protegida en México y utilizó, como marco analítico, el sistema de innovación agrícola. Las regiones analizadas fueron Puebla, Tlaxcala e Hidalgo. Los resultados indican que en las tres regiones estudiadas hay baja adopción de innovaciones y que los sistemas de innovación presentan una articulación débil y una tendencia de rentabilidad baja. Para consolidar la agricultura protegida como una alternativa para el desarrollo rural, es necesario implementar proyectos orientados al fortalecimiento de los sistemas de innovación que consideren las características territoriales. 


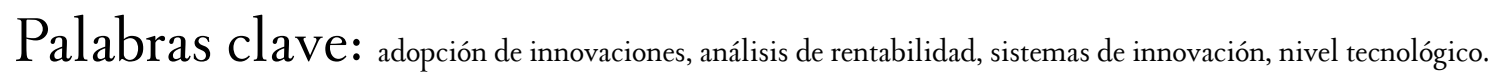

\title{
The Innovation System as an Analytical Framework for Protected Agriculture in the Mexican Central Region
}

\begin{abstract}
:
This research aims to analyze the protected agriculture in Mexico by using as an analytical framework the agricultural innovation system. The studied regions included Puebla, Tlaxcala and Hidalgo. The results indicate that these three regions have few cases of adopted innovations and that the innovation systems have a weak articulation as well as a low profitability trend. To consolidate the protected agriculture as an alternative in the rural development area, it is necessary to implement projects intended to strengthen innovation systems that consider the territorial characteristics.
\end{abstract}

Keywords: innovation adoption, profitability analysis, innovation systems, technological levelf.

\section{Introducción}

La agricultura protegida se define como el uso de toda estructura cerrada, cubierta por materiales transparentes o semitransparentes, que permite obtener condiciones artificiales de microclima para el cultivo de plantas (Santos, Obregón-Olivas y Salamé-Donoso, 20ro). De esta manera, es posible incrementar la cantidad, calidad y oportunidad comercial de los productos hortícolas (Bastida, 2008; Castañeda-Miranda, Ventura-Ramos, PenicheVera y Herrera-Ruiz, 2007; Moreno, Aguilar, y Luévano, 20II). Para alcanzar esos rendimientos potenciales y mantener una agricultura protegida sostenible, es necesario un conocimiento profundo de estos sistemas de producción (Vargas-Canales, Castillo-González, Pineda-Pineda, Ramírez-Arias y Avitia-García, 20I4).

Las ventajas que ofrece la agricultura protegida han incentivado a los agricultores a adoptar este sistema de producción, lo que ha acelerado su expansión a nivel global en las últimas dos décadas (Max et al., 20ı2; VargasCanales, Palacios-Rangel, Aguilar-Ávila y Ocampo-Ledesma, 2016). Se estima que la superficie mundial con invernaderos es de aproximadamente 750.000 ha. China es el país donde más rápido está creciendo, a un ritmo del 30\% anual (García Torrente y Pérez Mesa, 2012), mientras que Almería, en España, es la región con mayor concentración de invernaderos en el mundo (Aznar, 20II).

Los agricultores mexicanos no son ajenos a este fenómeno, pues en casi todas las regiones agrícolas continúan transitando de la producción a campo abierto a las tecnologías de agricultura protegida (Kuss, Flores y Harrison, 20I6). Aunada a lo anterior, la tecnología de agricultura protegida es uno de los sistemas más promovidos en los programas gubernamentales y es considerada como una estrategia viable para impulsar el crecimiento y la productividad en el sector agropecuario (Vargas-Canales, Palacios-Rangel, Aguilar-Ávila, Ocampo-Ledesma y Medina-Cuellar, 2018).

Los datos más recientes indican que, en el 2015, en México la superficie con agricultura protegida era de poco más de 51.000 ha (Secretaría de Agricultura, Ganadería, Desarrollo Rural, Pesca y Alimentación [Sagarpa] y Servicio de Información Agroalimentaria y Pesquera [SIAP], 2015), con un ritmo de crecimiento del $12 \%$ anual (Ramos 
Gourcy, I4 de mayo de 20I4). Los estados con una mayor superficie de agricultura protegida son Sinaloa, Baja California y Jalisco, y en menor medida les siguen estados como Colima, Estado de México, Hidalgo, Puebla, Tlaxcala, Oaxaca, Michoacán, Querétaro, San Luis Potosí, Sonora y Zacatecas, que presentan un buen ritmo de crecimiento.

De acuerdo con Kuss et al. (2016), esta tasa de crecimiento se debe principalmente al incremento de las exportaciones a EE. UU., al aumento de los precios internacionales y a la devaluación del peso mexicano, pero también a la alta incidencia de plagas y enfermedades, los altos costos de producción, la escasa disponibilidad de agua y a los fenómenos meteorológicos que afectan la producción a cielo abierto. Es decir, esta tecnología se adopta como una estrategia de administración de riesgos que utilizan los productores para minimizar las incertidumbres que enfrenta la producción agrícola.

De manera general, en México se pueden observar dos vertientes de la agricultura protegida; en la primera, se ubica el sistema empresarial y de alta tecnología del norte del país, vinculado directamente al mercado internacional, y en la segunda, que se ubica en el centro y el sur del país, predominan los pequeños productores y su vinculación con los mercados locales y regionales (Vargas-Canales et al., 20I6). Con respecto al nivel tecnológico, los niveles más bajos los tienen los pequeños agricultores, que además tienen canales deficientes de comercialización, capital de trabajo insuficiente, baja capacidad de hacer frente a los fenómenos meteorológicos y acceso limitado a créditos (Kuss et al., 2016).

Pese al rápido crecimiento de la agricultura protegida, diversas investigaciones académicas y reportes de asociaciones civiles indican que, en promedio, el 50\% de los invernaderos subsidiados por el Gobierno a través de la Sagarpa no están en producción I8 meses después de su puesta en marcha (Asociación Mexicana de Horticultura Protegida A. C. [Amhpac], 2009; García-Sánchez, Aguilar-Ávila y Bernal-Muñoz, 20II).

Los estudios en el área económica-social de la agricultura protegida en México, que han explicado esta problemática, han logrado importantes avances; por ejemplo, han analizado los factores que inciden en su funcionamiento (Moreno et al., 20II), la adopción de innovaciones y el nivel de equipamiento (García-Sánchez et al., 20II), la importancia de la confianza en los procesos de difusión y la adopción de innovaciones (VargasCanales, Palacios-Rangel, Camacho-Vera, Aguilar-Ávila y Ocampo-Ledesma, 2015), la evaluación económica de este sistema de producción (Rucoba, Anchondo, Luján y Olivas, 2006; Terrones Cordero y Sánchez Torres, 20II) y los modelos de transferencia de tecnología (Borbón Morales y Arvizu Armenta, 2015).

Sin duda, estos trabajos ayudan a comprender el comportamiento de la agricultura protegida en las regiones donde se ha introducido; sin embargo, se han elaborado de manera aislada y sin considerar que forman parte de un sistema de innovación interconectado a nivel regional. De ahí que, para entender el comportamiento de la agricultura protegida de la región centro del país, es necesario, además de estudiar y comparar sus principales características, analizar la configuración e interacción de los sistemas de innovación en los que está inmersa.

En este trabajo se estudia la agricultura protegida del centro de México, bajo un enfoque de sistema de innovación en el que se analizan las interacciones entre los actores encargados de generar las innovaciones y sus usuarios (Edquist, 200I; Lundvall, 1992). En ese sentido, Edquist (1997) y Carlsson, Jacobsson, Holmén y Rickne (2002) definieron los sistemas de innovación como todos los factores económicos, sociales, políticos y organizativos que se constituyen por la interacción que se establece entre todos los actores. Este marco analítico representa un cambio significativo en el enfoque lineal convencional que se tenía sobre el cambio tecnológico, pues enfatiza la importancia de estudiar un sistema de innovación como una unidad que comprende a todos los componentes involucrados en el proceso de innovación (Spielman, Davis y Negash, 20II).

Los atributos son las propiedades de los componentes, que se modifican gradualmente por efecto de la interacción entre estos, en condiciones de incertidumbre y a lo largo del tiempo (Lane y Maxfield, 1996). El estudio de los sistemas de innovación, al centrarse en los factores determinantes de la innovación y sus consecuencias, 
permite proponer estrategias que favorezcan el crecimiento económico, el aumento de la rentabilidad, la capacidad de generación de empleo, el desarrollo sustentable, etcétera.

En ese sentido, al pensar en los sistemas de innovación y en su influencia en el desarrollo rural, es importante considerar lo planteado por Galdeano-Gómez, Aznar-Sánchez y Pérez-Mesa (20II), quienes indican que existen múltiples trayectorias de desarrollo resultantes de diversas combinaciones de fuerzas locales, regionales, nacionales y globales en los territorios. De acuerdo con Vázquez-Barquero y Rodríguez-Cohard (2016), es necesario fortalecer las instituciones y fomentar la interacción de las fuerzas de desarrollo y la combinación de los objetivos de los diferentes actores vinculados a los sistemas de producción, para cambiar las ventajas comparativas en ventajas competitivas.

Derivado de lo anterior, los esquemas de desarrollo colectivo deben considerar las características e interacciones que se desarrollan y evolucionan en los territorios, con el fin de diseñar una política integral que estimule la coordinación de los diferentes actores (económicos, políticos y sociales) a través de la combinación de sus objetivos e intereses, para promover el cambio tecnológico, la innovación y el desarrollo económico sostenido (VargasCanales et al., 2018). Por consiguiente, los sistemas de innovación y su activación son los instrumentos ideales para impulsar el desarrollo local, ya que surgen, evolucionan y se reconfiguran constantemente de acuerdo con la dinámica económica (Caravaca, González, García, Fernández y Mendoza, 20I4; Hekkert, Suurs, Negro, Kuhlmann y Smits, 2007; Organización para la Cooperación y el Desarrollo Económicos [OCDE], 2004; Spielman, Ekboir, Davis y Ochieng, 2008).

En el sector agrícola, los sistemas de innovación son el resultado de un proceso social coevolutivo de las interacciones que se desarrollan en los territorios y están influenciados por las condiciones geográficas, económicas y políticas (Vargas-Canales et al., 2016). Los anteriores argumentos son la base del presente trabajo, que plantea como hipótesis que los sistemas de innovación, que se han configurado entorno a la agricultura protegida en la región centro de México, presentan una débil interacción, lo que limita el flujo de recursos, el conocimiento y la información, junto con la capacidad de los agricultores para mantenerse en el mercado.

En ese sentido, esta investigación tuvo como objetivo general analizar la agricultura protegida utilizando como marco analítico el sistema de innovación agrícola (sus componentes, relaciones e interacciones y funcionalidad) en Puebla, Tlaxcala e Hidalgo, México. Para esto, se plantearon los siguientes objetivos específicos: I) describir el perfil de los agricultores y de las unidades de producción, 2) estimar la adopción de innovaciones e identificar el nivel tecnológico de los invernaderos, 3) analizar los sistemas de innovación para la agricultura protegida en la región centro de México, 4) analizar los canales de comercialización de la agricultura protegida y 5) estimar el desempeño del sistema de innovación mediante el cálculo de la rentabilidad de esta actividad.

\section{Metodología}

\section{Regiones de estudio}

La investigación se realizó en tres regiones geográficas del centro de México donde se desarrollan procesos de reconversión productiva y tecnológica, para pasar de la agricultura a cielo abierto a la agricultura protegida. Las regiones analizadas fueron: Aquixtla, en Puebla, la región noroeste de Tlaxcala y la región de Tulancingo, en Hidalgo (tabla I). Estas regiones están integradas por varios municipios y en ellas se tiene la mayor concentración de agricultura protegida. En las tres regiones se encontró que el 98\% de los agricultores entrevistados producen jitomate (Solanum lycopersicum L.). 
TABLA 1

Características de las Regiones anAlizadas

\begin{tabular}{llcc}
\hline Región & Clima & T $\left({ }^{\circ} \mathbf{C}\right) / \mathbf{P}(\mathbf{m m})^{*}$ & SAP (ha) ${ }^{* *}$ \\
\hline Puebla & $\begin{array}{l}\text { Predomina el templado subhúmedo con } \\
\text { 1luvias en verano }\end{array}$ & $12-18 / 600-900$ & 70 \\
Tlaxcala & $\begin{array}{l}\text { Dos climas principales; uno moderadamente } \\
\text { frío, con régimen de lluvias en verano, y otro } \\
\text { templado subhúmedo, con lluvias en verano }\end{array}$ & $2-16 / 500-1100$ & 20 \\
& & & \\
Hidalgo & Templado subhúmedo con lluvias en verano & $12-16 / 500-900$ & 80 \\
\hline
\end{tabular}

Fuente: Instituto Nacional de Estadística y Geografía (Inegi) (2009)

* Rangos de temperatura $(\mathrm{T})$ y precipitación $(\mathrm{P})$

** Superficie con agricultura protegida, de acuerdo con informantes clave y productores entrevistados

\section{Población y muestra}

En esta investigación se utilizó un muestreo no estadístico, que, para este caso, fue el muestreo con combinaciones de técnicas intencionales o de conveniencia con propósito múltiple. Este método, que consiste en combinaciones de dos o más técnicas de muestreo, es conveniente cuando se busca seleccionar distintos grupos focales que puedan aportar más y mejor información. La aplicación de dos o más técnicas permite desarrollar investigaciones más completas y pertinentes (Teddlie y Yu, 2007).

Para estimar los indicadores de adopción de innovaciones y rendimiento, se seleccionaron agricultores que han recibido subsidios públicos para la construcción de invernaderos, con base en el padrón de las secretarías estatales de desarrollo rural. Para identificar la configuración del sistema de innovación para la agricultura protegida, se entrevistó a distintos actores (tabla 2) seleccionados por el método de bola de nieve (Goodman, 196I). Finalmente, para desarrollar el estudio de la rentabilidad de la actividad, se combinó la técnica de paneles de expertos o grupos focales (Hamui-Sutton y Varela-Ruiz, 20I3) y la construcción de unidades representativas de producción (Sagarnaga et al., 1999). 
TABLA 2

TIPO Y NÚMERO DE ACTORES SELECCIONADOS POR REGIÓN

\begin{tabular}{lccc}
\hline Tipo de actor & Puebla & Tlaxcala & Hidalgo \\
\hline Estimación de indicadores & 42 & 25 & 58 \\
Agricultores & 50 & 30 & 50 \\
Porcentaje de la población & & & \\
Análisis del sistema de innovación & 5 & 3 & 6 \\
Agricultor líder & 3 & 2 & 5 \\
Asesor técnico & 2 & 1 & 1 \\
Funcionario municipal & 1 & 1 & 2 \\
Funcionario estatal & 1 & 1 & 1 \\
Funcionario federal & 3 & 2 & 3 \\
Proveedor & 1 & 1 & 2 \\
Comercializador & 1 & 1 & 1 \\
Agente financiero & 1 & 1 & $\mathbf{8 0}$ \\
Investigación y desarrollo & $\mathbf{6 0}$ & $\mathbf{3 8}$ & \\
Total & &
\end{tabular}

Fuente: elaboración propia

\section{Instrumentos para la colecta de información}

\section{Características de las unidades de producción y adopción de innovación}

Para recolectar datos e información, se diseñó un instrumento que se aplicó a través de una encuesta semiestructurada dividida en cuatro secciones: sección I: perfil de los agricultores (nombre, ubicación, edad, escolaridad y experiencia en agricultura protegida); sección 2: perfil de las unidades de producción (tamaño de la unidad de producción en $\mathrm{m}^{2}$, rendimiento en $\mathrm{kg} / \mathrm{m}^{-2}$ y características tecnológicas); sección 3 : lista de innovaciones que los agricultores deben adoptar para alcanzar la sustentabilidad en la producción de jitomate en invernadero y para aprovechar las ventajas de la agricultura protegida (la cuarta sección se explica más abajo).

Estas secciones se agruparon de acuerdo con las categorías propuestas en el Manual de Oslo (OCDE, 2005), a saber: I) organización, 2) proceso, 3) producto y 5) mercado. Para reconocer una innovación, esta debe cumplir con el hecho de ser nueva para la unidad de producción (OCDE, 2005); es decir, una innovación pudo haber sido introducida ya por otros agricultores, pero si es nueva para la empresa entrevistada (o significativamente mejorada), se considera una innovación. 


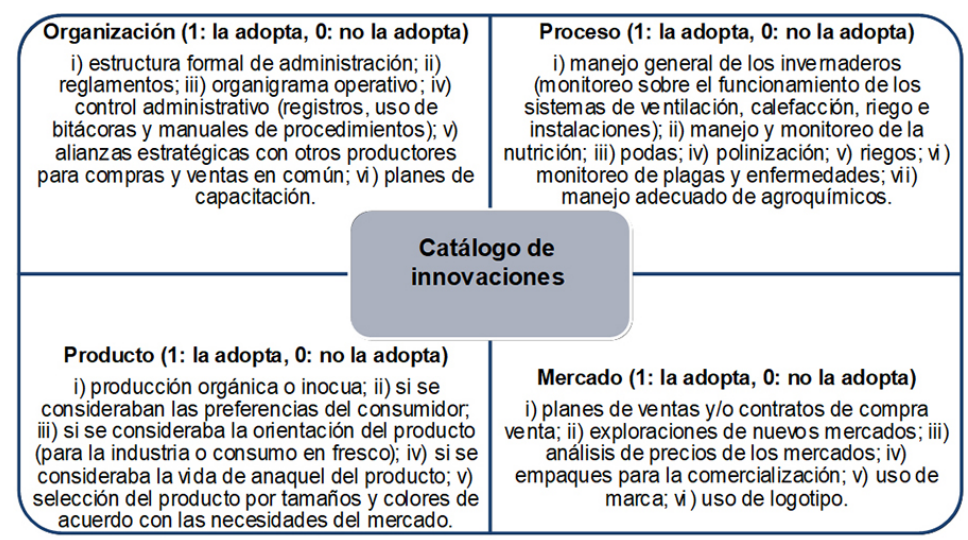

FIGURA 1

LiSTADO DE INNOVACIONES QUE FUERON VERIFICADAS POR CATEGORÍA

Fuente: elaboración propia con base en datos de campo y en Vargas-Canales et al. (2018)

El listado de innovaciones (figura I) se construyó y validó en entrevistas y talleres participativos con agricultores y asesores técnicos profesionales, así como con investigadores de reconocido prestigio en las regiones de estudio. En concordancia con Muñoz-Rodríguez, Rendón-Medel, Aguilar-Ávila, García-Muñiz y Altamirano-Cárdenas (2004), en los hechos, cada una de las prácticas a verificar puede registrar tantas variaciones específicas como agricultores existentes, y, siguiendo a Muñoz-Rodríguez et al. (2004), este método mide la proporción de innovaciones adoptadas por cada agricultor entrevistado con respecto al total. Así, el resultado es el índice de adopción de innovaciones (INAI) por agricultor y por categoría (tabla 3 ).

TABLA 3

FóRMULAS PARA EL CÁLCULO DE INDICADORES DE ADOPCIÓN DE INNOVACIONES

\begin{tabular}{|c|c|c|}
\hline Indicador & Fórmula & En la cual \\
\hline \multirow{3}{*}{$\begin{array}{l}\text { Índice de adopción de } \\
\text { innovaciones para cada } \\
\text { agricultor }\end{array}$} & $\begin{array}{l}\text { INAI } I_{i k} \\
\sum_{j=1}^{n} \text { Innov }_{j k}\end{array}$ & $\begin{array}{l}I N A I_{k}=\text { índice de adopción del i- } \\
\text { ésimo agricultor }\end{array}$ \\
\hline & $=\frac{n}{n}$ & $\begin{array}{l}\text { Innov }_{j k}=\mathrm{j} \text {-ésima innovación } \\
\text { verificada }\end{array}$ \\
\hline & & $n=$ número total de innovaciones \\
\hline \multirow{3}{*}{$\begin{array}{l}\text { Índice de adopción de } \\
\text { innovaciones por categoría } \\
\text { (organización, proceso, } \\
\text { producto y mercado) para } \\
\text { cada agricultor }\end{array}$} & $\operatorname{IAIC}_{i}=\frac{\sum_{j=1}^{n} I N A I_{k}}{k}$ & $\begin{array}{l}L A I C_{i k}=\text { índice de adopción del i- } \\
\text { ésimo agricultor en la k-ésima } \\
\text { categoría }\end{array}$ \\
\hline & & $\begin{array}{l}I N A I_{k}=\mathrm{k} \text {-ésima innovación } \\
\text { verificada de la } \mathrm{k} \text {-ésima categoría }\end{array}$ \\
\hline & & $\begin{array}{l}k=\text { número total de innovaciones en } \\
\text { la } \mathrm{k} \text {-ésima categoría }\end{array}$ \\
\hline
\end{tabular}

Fuente: elaboración propia

Finalmente, en la sección 4 se mapeó la red comercial, y para ello se identificaron a) las empresas o personas proveedoras de insumos y equipos necesarios para la producción, el tipo de insumo o equipo y su ubicación, y b) las empresas o personas a las que se les vende la producción, junto con el tipo de agente (mayorista, minorista, consumidor final...) y su ubicación. 


\section{Sistema de innovación}

Para el análisis del funcionamiento del sistema de innovación, es decir, de cómo y para qué interactúan los diferentes actores involucrados en la producción, generación y difusión de conocimiento y del rol de los agentes de Gobierno, los financiadores y comercializadores, se construyó un cuestionario guía para colectar información respecto a estos actores que se relacionan con el sistema de producción de jitomate en la agricultura protegida.

\section{Rentabilidad}

La obtención de datos de ingresos y egresos de las unidades de producción agropecuaria ha representado una limitante en los estudios de rentabilidad (Ruiz, Ruiz, Torres y Cach, 20I2). En este caso, para obtener los datos, se utilizó la técnica de paneles de expertos o grupos focales (Hamui-Sutton y Varela-Ruiz, 2013), en los que se construyeron unidades representativas de producción (URP). Una URP tipifica las actividades y decisiones de los agricultores participantes en un panel o grupo focal y representa una unidad de producción característica de una escala y un sistema de producción particular de una región productora (Elliott, 1928; Sagarnaga et al., 1999).

Este método ha sido adaptado para estudiar los impactos económicos a nivel de empresas agropecuarias en Estados Unidos desde 1996 (Smith et al., 1997). Las URP son una herramienta útil para obtener información económica confiable, precisa, contextualizada y a bajo costo (Sagarnaga et al., 1999). Por otra parte, para la integración y el desarrollo de los paneles de expertos, se utilizó el procedimiento metodológico propuesto por Sagarnaga et al. (1999).

Con los datos obtenidos se estimaron los costos financieros de producción en que incurren los agricultores; los costos financieros incluyen todos los costos fijos y variables, pero no el costo de gestión empresarial, ni la mano de obra del productor o familiar (si estas no son remuneradas explícitamente), ni el costo de oportunidad del capital invertido en gastos de operación o en activos fijos como la tierra, las construcciones, las instalaciones, la maquinaria y el equipo (Agricultural \& Applied Economics Association [AAEA], 2000). Para estimar la rentabilidad de las unidades de producción, se calculó la relación beneficio-costo, y de esta manera, la porción que exceda a la unidad indicará el grado de rentabilidad de la actividad (Gittinger, 1983).

\section{Resultados}

\section{Perfil de productores}

Los resultados indican que los agricultores dedicados a la agricultura protegida en las regiones analizadas se encuentran es su madurez productiva y son más jóvenes con respecto a los que se dedican a otras actividades agropecuarias, particularmente en Hidalgo (tabla 4). En promedio, se tienen productores de 5r años de edad, con una experiencia en el manejo de la agricultura protegida que oscila entre 5 y io años en promedio. Es importante mencionar que cada año transitan más productores a la agricultura protegida, y esto hace que la media se mantenga baja. Con respecto al nivel de escolaridad, el promedio se ubica en io años y no presenta gran variación. Resalta, en este caso, la región de Tlaxcala, donde se tiene un grupo importante de profesionales que se dedican a esta actividad. 
TABLA 4

EsTADíSTICOS DESCRIPTIVOS DEL PERFIL DE PRODUCTORES DE JITOMATE

en agricultura protegida en Puebla, Tlaxcala e Hidalgo, México

\begin{tabular}{lcccc}
\hline Entidad & $\begin{array}{l}\text { Número de } \\
\text { productores }\end{array}$ & Edad & Experiencia & Escolaridad \\
& & \multicolumn{3}{c}{ (Promedio en años) } \\
\hline Puebla & 25 & 54,2 & 9,6 & 8,9 \\
Tlaxcala & 42 & 57,1 & 10,4 & 12 \\
Hidalgo & 58 & 42,1 & 5,26 & 9,1 \\
Promedio & & 51,1 & 8,4 & 10 \\
Coeficiente de variación & & 39,6 & 15,6 & 32,8 \\
\hline
\end{tabular}

Fuente: elaboración propia con base en datos de campo

\section{Unidades de producción y adopción de innovaciones}

Las unidades de producción que prevalecen en las regiones analizadas son pequeñas y, en promedio, no superan los $2000 \mathrm{~m}^{2}$ (tabla 5). Las superficies menores se encuentran en Tlaxcala y Puebla. Para el caso de Hidalgo, se presentan superficies de tamaños más grandes, y esto se debe a la ampliación que se ha dado a través del tiempo de las unidades de producción. Lo anterior sugiere que la región de Hidalgo presenta un ritmo mayor de crecimiento de la agricultura protegida en comparación con Tlaxcala y Puebla.

TABLA 5

Estadísticos DESCR IPTIVOS DEL PERFIL DE LAS UNIDADES DE PRODUCCIÓN DE jitomate en agricultura protegida en Puebla, Tlaxcala e Hidalgo, México

\begin{tabular}{lcccc}
\hline Entidad & Número $^{*}$ & $\begin{array}{c}\text { Superficie } \\
\left(\mathbf{m}^{\mathbf{2}}\right)\end{array}$ & Rendimientos $\left(\mathbf{k g} / \mathbf{m}^{\mathbf{2}}\right)$ & INAI \\
\hline Puebla & 25 & 1250 & 28,10 & 0,60 \\
Tlaxcala & 42 & 972 & 12,82 & 0,33 \\
Hidalgo & 58 & 3463 & 21,43 & 0,58 \\
Promedio general & & 1895 & 20 & 0,50 \\
Coeficiente de variación & & 39,6 & 7 & 36,9 \\
\hline
\end{tabular}

Fuente: elaboración propia con base en datos de campo

* Número de unidades de producción 
Con respecto a los rendimientos obtenidos, se observan fuertes variaciones. Los rendimientos más altos se tienen en Puebla, seguida de Hidalgo, y Tlaxcala presenta los rendimientos más bajos. Esto se debe al manejo de los sistemas de producción y a la introducción de innovaciones tecnológicas, pues el tipo de estructura, el método de control del clima y el manejo de cultivo son similares en las tres regiones (figura 2).

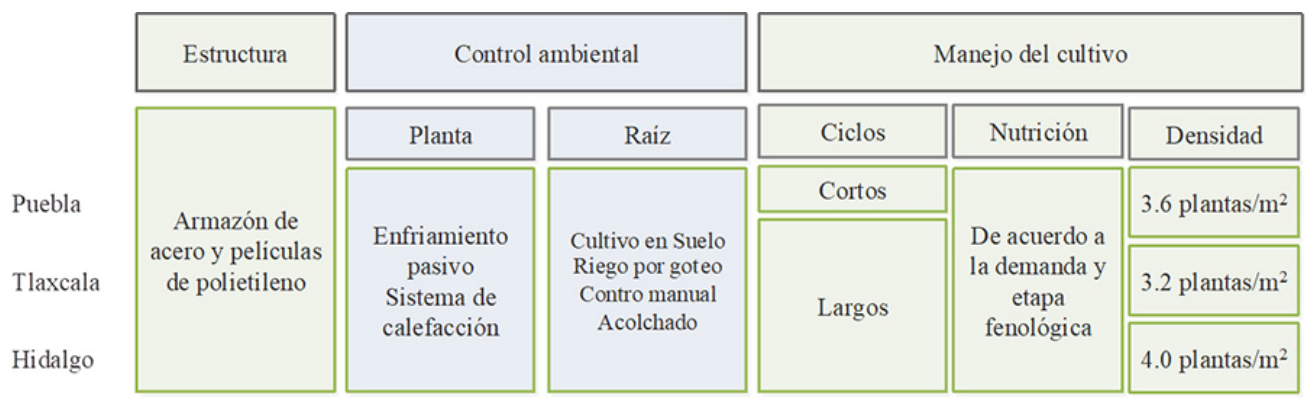

FIGURA 2

Componentes de los niveles tecnológicos de la PRoducción de Jitomate en agricultura protegida en Puebla, Tlaxcala e Hidalgo, México

Fuente: elaboración propia con base en datos de campo y en Costa y Giacomelli (2005)

Con relación al INAI, las regiones de Puebla e Hidalgo presentan un comportamiento similar, mientras que en Tlaxcala se presentan los niveles más bajos (tabla 5). Las innovaciones que se adoptan con mayor frecuencia son las de proceso, seguidas de las de organización, producto y mercado (figura 3). Sin embargo, el promedio general

es muy bajo (tabla 5), lo que indica que la adopción de innovaciones es deficiente e insuficiente para alcanzar rendimientos cercanos al potencial, lo que hace necesario que se implementen estrategias que permitan mejorar y llevar el sistema de producción a su máximo potencial productivo.

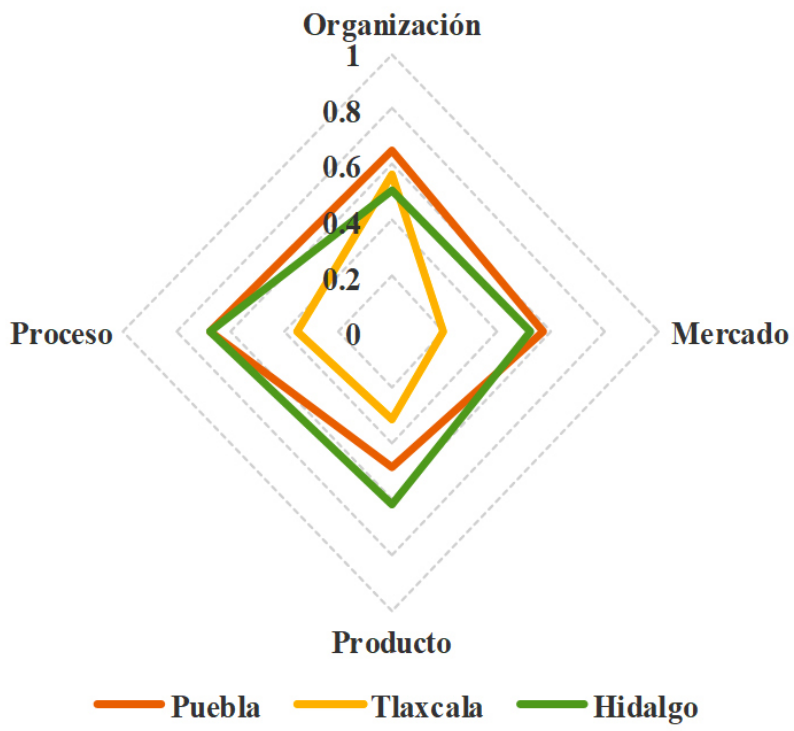

FIGURA 3

ÍNDICE DE ADOPCIÓN DE INNOVACIONES DE LA PRODUCCIÓN DE JITOMATE EN agricultura protegida en Puebla, Tlaxcala e Hidalgo, México, por categoría

Fuente: Elaboración PROPIA CON BASE EN DATOS DE CAMPO 
En la categoría de proceso se presentan los niveles más altos, debido a que son las innovaciones que se relacionan con el manejo de los invernaderos y del cultivo. En este sentido, en Puebla e Hidalgo se tienen los INAI más altos (tabla 5), lo que permite inferir que los agricultores tienen un mejor dominio del proceso de producción, y esta situación está relacionada con mayores rendimientos.

Respecto a la categoría de organización, los mayores niveles los presentan Puebla y Tlaxcala, lo que muestra que los agricultores son más propensos a aceptar planteamientos relacionados con la acción colectiva. En relación con la categoría de producto, se destacan Hidalgo y Puebla, lo que revela que estos agricultores implementan estrategias de diferenciación de su producto y tienen en cuenta las preferencias de los consumidores. Con relación a la adopción de innovaciones de mercado, los productores de Puebla e Hidalgo adoptan un mayor número de prácticas, lo que indica que se mantienen informados sobre el comportamiento de los mercados (figura 3 ).

\section{Nivel tecnológico}

Los sistemas de producción en agricultura protegida de las tres regiones analizadas son similares. De manera general, se caracterizan por tener estructuras de acero galvanizado cubiertas de polietileno, mallas antiáfidos y sistemas de control pasivo, además de que se usa el suelo como sustrato (figura 3). Aunque la infraestructura es similar, se presentan diferencias en los procesos de producción: en Puebla, se tienen ciclos de producción de 6 meses, y en Hidalgo, la densidad es de 4 plantas por $\mathrm{m}^{2}$. Para el caso de Puebla, la principal fuente de abastecimiento de agua son ríos y manantiales, mientras que para Tlaxcala e Hidalgo la fuente de agua son los pozos profundos.

\section{Sistemas de innovación}

Los resultados revelan que los sistemas de innovación en las tres regiones se encuentran en una etapa de arranque y están caracterizados por: I) una débil interacción de los actores del sistema de educación e investigación con el resto de los actores del sistema, 2) organizaciones de Gobierno que focalizan sus recursos en el subsidio de activos, 3 ) escasa participación de agentes que articulan a los diferentes actores, en este caso asesores técnicos o extensionistas, y 4) especificaciones de calidad determinadas por los intermediarios y el tipo de mercado que abastecen (figura 4). Es importante mencionar que en las tres regiones se destacan como actores importantes los proveedores de insumos, pues son una fuente importante de información e innovación. En conjunto, se convierten en agentes que desempeñan funciones de transferentes de tecnología de ciclo corto.

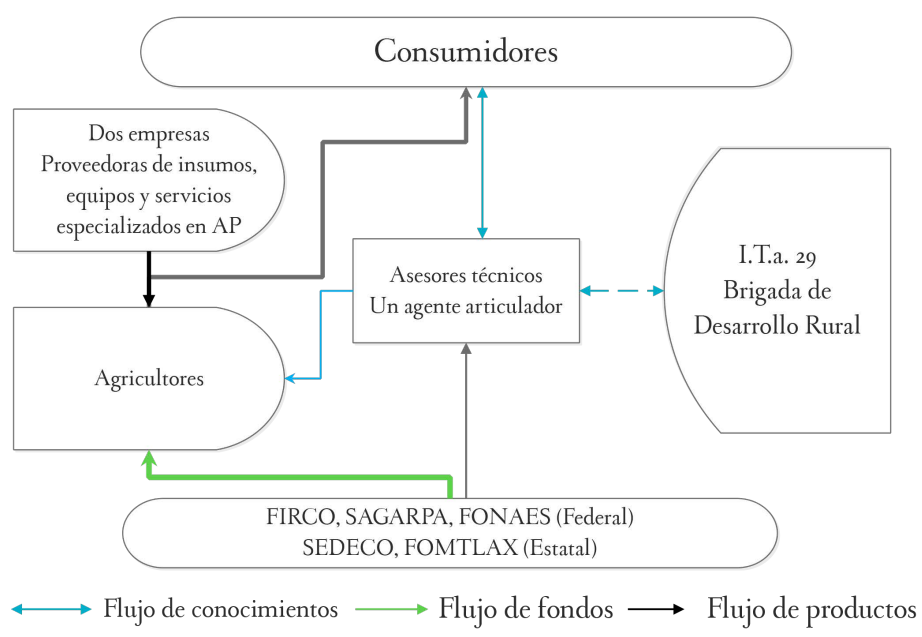

FIGURA 4A. TLaxcala 


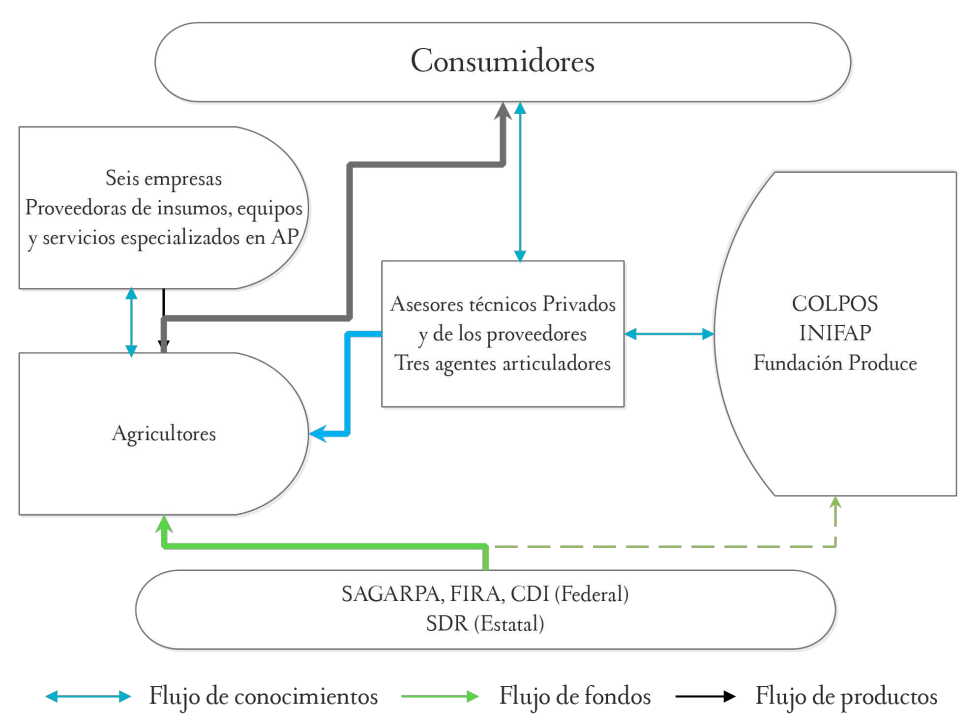

FIGURA 4в. Puebla

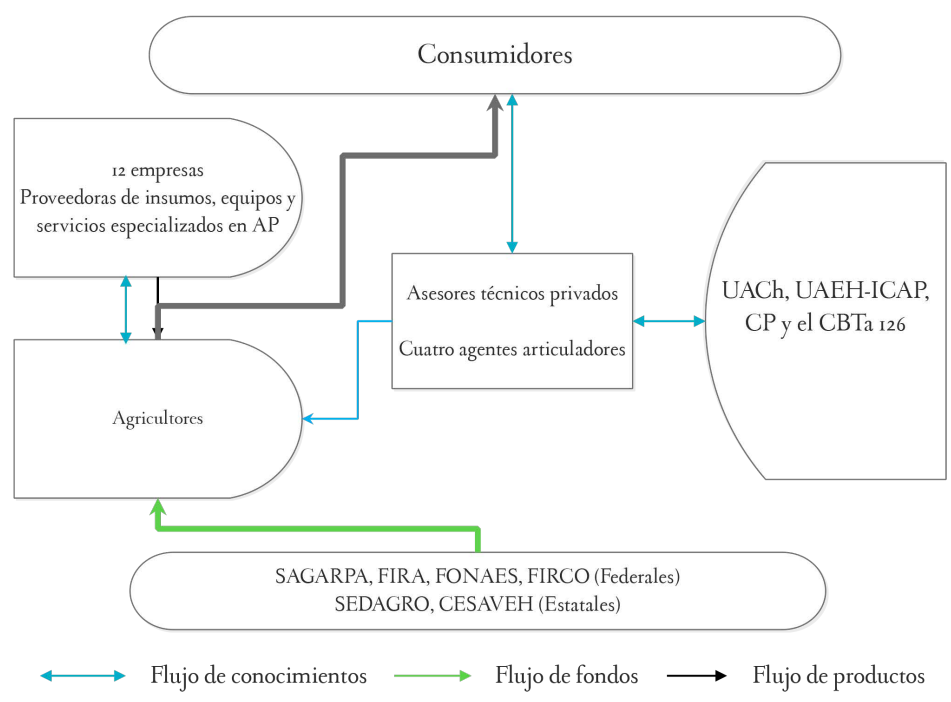

FIGURA 4c. HidAlgo

FIGURA 4 (A, B Y C)

Componentes DEL SISTEMA DE INNOVACIÓN DE LA PRODUCCIÓN DE Jitomate en agricultura protegida en Tlaxcala, Puebla e Hidalgo , México

* La intensidad de las líneas indica la intensidad de la interacción

Inifap: Instituto Nacional de Investigaciones Forestales, Agrícolas y Pecuarias; Sagarpa: Secretaría de Agricultura, Ganadería, Desarrollo Rural, Pesca y Alimentación; FIRA: Fideicomisos Instituidos en Relación con la Agricultura; Fonaes: Fondo Nacional de Apoyo a Empresas de Solidaridad; Firco: Fideicomiso de Riesgo Compartido; Sedagro: Secretaría de Desarrollo Agropecuario; Cesaveh: Comité Estatal de Sanidad Vegetal de Hidalgo; UACh: Universidad Autónoma Chapingo; UAEH-ICAP: Universidad Autónoma del Estado de Hidalgo;

ICAP: Instituto de Ciencias Agropecuarias; CP: Colegio de Postgraduados; ITA 29: Instituto Tecnológico Agropecuario n. ${ }^{\circ}$ 29; CBTA I26: Centro de Bachillerato Tecnológico Agropecuario n. ${ }^{\circ}$ i26; Sedeco: Secretaría de Desarrollo Económico; Fomtlax: Fondo Macro para el Desarrollo Integral de Tlaxcala; CDI: Comisión Nacional para el Desarrollo de los Pueblos Indígenas; SDR: Secretaría de Desarrollo Social.

Fuente: ELABORACión PROPIA CON BASE EN DATOS DE CAMPO 
Los principales centros de investigación y universidades que se vinculan con la agricultura protegida, que son quienes difunden, capacitan y desarrollan investigaciones, son los siguientes: en la región de Puebla, el Colegiode Postgraduados, la Fundación Produce Puebla y el Instituto Nacional de Investigaciones Forestales, Agrícolas y Pecuarias; en Tlaxcala, el Instituto Tecnológico Agropecuario n. ${ }^{\circ}$ I29, y en Hidalgo, se vinculan de forma más activa la Universidad Autónoma del Estado de Hidalgo (el Instituto de Ciencias Agropecuarias y el Instituto de Ciencias Económico-Administrativas) y la Universidad Autónoma Chapingo (el Departamento de Fitotecnia y el Centro de Investigaciones Económicas, Sociales y Tecnológicas de la Agroindustria y la Agricultura Mundial) (figura 4).

\section{Canales de comercialización}

El mercado se caracteriza por un gran número de oferentes de jitomate tipo saladette homogéneo en tamaño y color, ubicados en las centrales de abasto de Huixcolotla (Puebla), Iztapalapa (Ciudad de México) y Tulancingo (Hidalgo). En los tres casos, prevalecen tres esquemas de comercialización: el primero y el más común se caracteriza por la venta directa y a granel a los mayoristas; el segundo y menos común se da mediante la venta directa a pequeños intermediarios ubicados en los mercados locales y regionales, y un tercer esquema, mucho menos frecuente, es la venta directa a las amas de casa (figura 5).

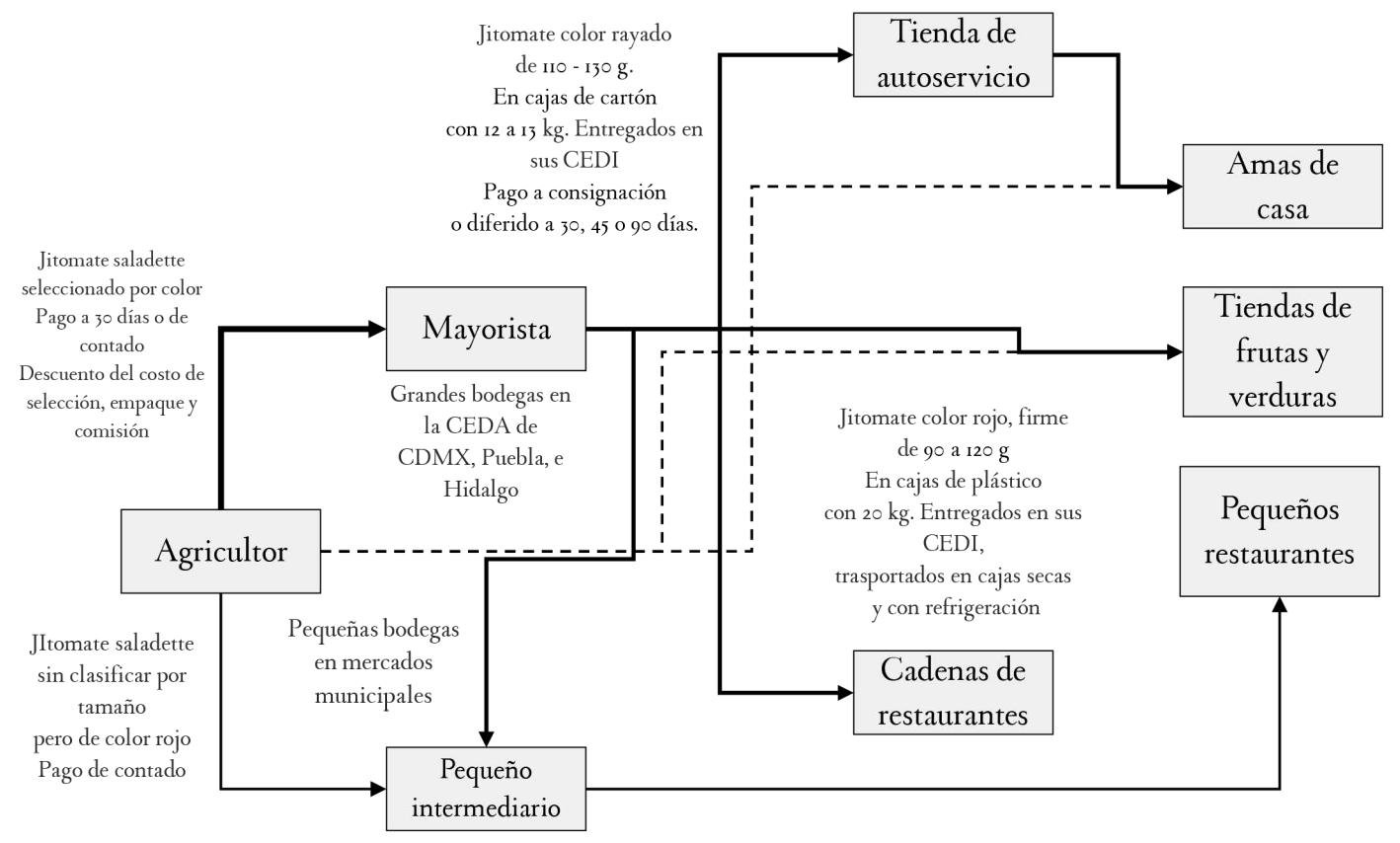

FIGURA 5

Canales de comercialización de jitomate en agricultura protegida en Puebla, Tlaxcala e Hidalgo, México Nota: El grosor de la línea indica la importancia del canal de distribución CEDA: Central de Abastos; CEDI: Centros de Distribución

FUENTE: ELABORACIÓN PROPIA CON BASE EN DATOS DE CAMPO 
Otro aspecto que afecta directamente la producción del centro del país es la producción en otros estados y el comportamiento del mercado internacional. En ese sentido, la producción a nivel nacional presenta un crecimiento constante; sin embargo, las exportaciones han aumentado a un ritmo mayor y el consumo nacional aparente presenta una tendencia negativa. Lo anterior indica que este es un producto altamente vinculado al comercio internacional y que pequeños cambios presentan grandes efectos a nivel local. Por otra parte, se destaca la disminución del consumo nacional aparente, lo que agudiza la problemática antes mencionada (figura 6).

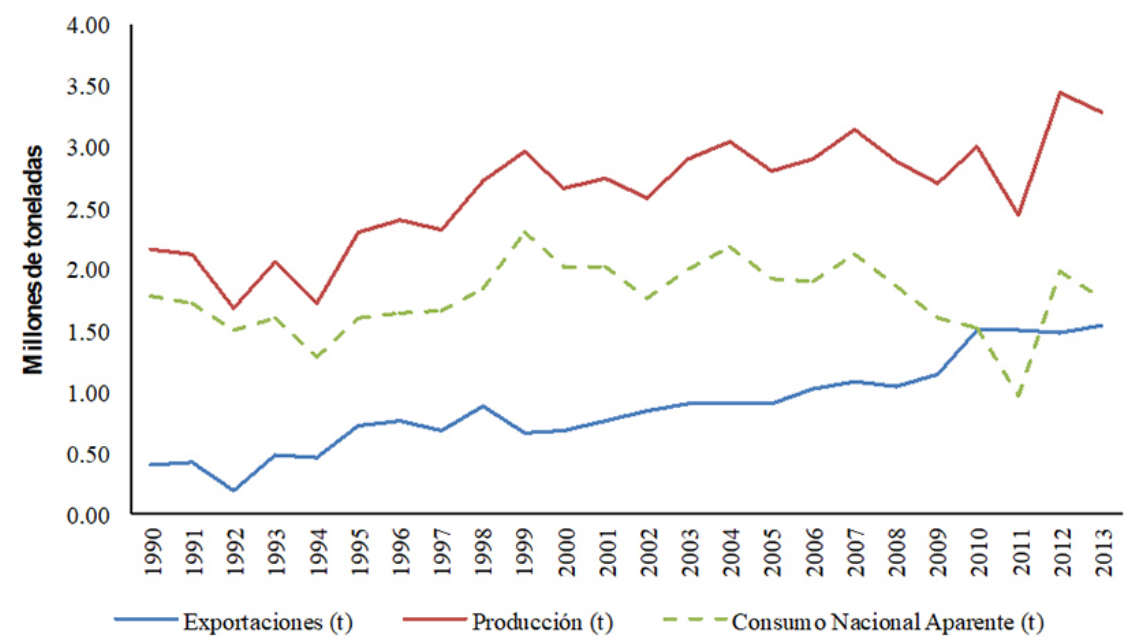

FIGURA 6

Producción, EXPORTACIONES Y CONSUMO NACIONAL APARENTE DE JITOMATE ENTRE LOS AÑos 1990 y 2013

Fuente: elaboración Propia con base en Sagar pa-SIAP (2013)

\section{Análisis de rentabilidad}

La estructura de costos presenta ligeras variaciones en las URP analizadas (tabla 6). Por ejemplo, la diferencia en costos de agroquímicos y combustibles se debe a las condiciones climáticas de Hidalgo, que tiene periodos más largos de lluvia y una oscilación térmica más intensa, lo que favorece las condiciones para la incidencia de enfermedades y mayor riesgo de bajas temperaturas, con lo que se aumenta la inversión en agroquímicos y calefacción. En los costos fijos no se presentan variaciones (tabla 6). Por otra parte, las depreciaciones presentan variaciones atribuibles al tipo y la calidad de los materiales de la estructura y la construcción. 
Análisis de rentabilidad de la producción de jitomate en agricultura protegida en Puebla, Tlaxcala e Hidalgo, México

\begin{tabular}{|c|c|c|c|}
\hline \multirow{2}{*}{ Conceptos } & \multicolumn{3}{|c|}{ Región } \\
\hline & Puebla & Tlaxcala & Hidalgo \\
\hline \multicolumn{4}{|c|}{ Costos variables (peso mexicano $/ \mathrm{m}^{2}$ ) } \\
\hline Fertilizantes & 18,16 & 19,71 & 21,56 \\
\hline Agroquímicos & 3,73 & 4,50 & 11,67 \\
\hline Plántula & 8,14 & 9,48 & 8 \\
\hline Accesorios & 2,21 & 3,12 & 1,90 \\
\hline Combustibles y lubricantes & 1,20 & 1,50 & 10,07 \\
\hline Herramientas & 0,38 & 0,43 & 0,67 \\
\hline Mantenimiento y reparaciones & 10,33 & 12,25 & 1,67 \\
\hline Mano de obra contratada & 21 & 24 & 26,40 \\
\hline Total de costos variables & 65,15 & 74,99 & 81,94 \\
\hline \multicolumn{4}{|l|}{ Costos fijos (peso mexicano/m²) } \\
\hline Impuestos & 0,40 & 0,43 & 0,43 \\
\hline Otros costos fijos & 0,90 & 1,10 & 0,80 \\
\hline Total de costos fijos & 1,30 & 1,53 & 1,23 \\
\hline \multicolumn{4}{|c|}{ Depreciaciones (peso mexicano/m²) } \\
\hline Estructura y sistema de riego & 34,84 & 36,16 & 39,58 \\
\hline Total de depreciaciones & 34,84 & 36,16 & 39,58 \\
\hline \multicolumn{4}{|l|}{ Otros (peso mexicano/m²) } \\
\hline Retiros de la URP & 36 & 40 & 32 \\
\hline Total de otros costos & 36 & 40 & 32 \\
\hline Rendimientos $\left(\mathrm{kg} / \mathrm{m}^{2}\right)$ & 30 & 28 & 28 \\
\hline Costo de producción por $\mathrm{m}^{2}$ & 137,29 & 152,68 & 154,75 \\
\hline Costo de producción por $\mathrm{kg}$ & 4,58 & 5,45 & 5,53 \\
\hline Precios de venta por $\mathrm{kg}$ & 7,50 & 7 & 6 \\
\hline Relación beneficio-costo & 1,64 & 1,28 & 1,09 \\
\hline
\end{tabular}

Fuente: elaboración propia con base en datos de campo

La relación beneficio-costo (B-C) indica que la agricultura protegida es una actividad viable en los tres casos. Es importante mencionar que los rendimientos obtenidos en las tres regiones son homogéneos, sin embargo, los precios de venta presentan variaciones de acuerdo con la región. Para el caso de Hidalgo, se debe mejorar el 
manejo del cultivo, lo que tendrá como consecuencia un aumento en el rendimiento y la reducción de sus costos de producción.

\section{Discusión}

\section{Perfil de productores}

Respecto a la edad de los productores, se tiene menores edades en comparación con productores dedicados a otras actividades del sector agropecuario, en el cual cerca del 70\% de los agricultores mexicanos tienen 60 años o más (Inegi, 2007; Organización de las Naciones Unidas para la Agricultura y la Alimentación [FAO] y Sagarpa, 20I4). Lo anterior, de acuerdo con Ramírez Valverde, Ramírez Valverde, Juárez Sánchez y Cesin Vargas (2007), se debe a la migración, pues los jóvenes prefieren actividades productivas alternativas mejor remuneradas que las del sector primario (García López y García Moreno, 2010).

Los resultados indican que la agricultura protegida representa una alternativa viable para los agricultores jóvenes, quienes ven en esta actividad un menor riesgo ante el entorno climático y la obtención de mejores utilidades. Además, los agricultores más jóvenes se encuentran en una etapa de búsqueda constante de mejores alternativas de desarrollo y tienen menor aversión al riesgo y una mejor actitud ante la adopción de innovaciones. Lo anterior concuerda con lo planteado por Aguilar-Gallegos, Muñoz-Rodríguez, Santoyo-Cortés y Aguilar-Ávila (2013a), quienes afirman que los agricultores con mayor edad tienen una menor propensión a adoptar innovaciones.

En lo que respecta a la escolaridad, el promedio de escolaridad formal es superior a la de productores dedicados a otras actividades del sector, de acuerdo con lo reportado por el Inegi y la Sagarpa (2015), que reportan que casi el $75 \%$ de los agricultores tiene seis o menos años de escolaridad en promedio. Lo anterior facilita la puesta en marcha de estrategias de desarrollo, debido a que la escolaridad tiene una relación positiva con la adopción de innovaciones o nuevas técnicas o prácticas de producción (Aguilar-Gallegos et al., 20rza; Perera, Sivayoganathan y Wijeratne, 2003; Ramírez Valverde et al., 2007; Sakib e Islam Afrad, 20I4).

Respecto a la experiencia en el manejo de este sistema de producción, dado que este es de reciente inserción en las tres regiones (en comparación con sistemas de producción tradicionales como el maíz, la cebada o el trigo), los agricultores entrevistados tienen poca experiencia en la actividad. Derivado de lo anterior, resulta pertinente implementar acciones de gestión de innovación para el desarrollo de sus capacidades técnico-productivas que les permitan superar la falta de experiencia sobre el manejo del sistema de producción. Es conveniente mencionar que, de acuerdo con las características de estos grupos, se infiere que la probabilidad de éxito de implementar una estrategia de intervención orientada al desarrollo regional es mayor con respecto a los sistemas tradicionales.

\section{Unidades de producción y adopción de innovaciones}

En cuanto a rendimientos, los resultados indican que la tecnología no está siendo aprovechada en su máximo potencial, por lo que es necesario identificar las innovaciones que permitan obtener los máximos rendimientos y aprovechar eficientemente las ventajas que ofrece este sistema de producción. Es conveniente decir que, a nivel 
nacional, se tiene una importante variación en cuanto a rendimientos; sin embargo, el comportamiento de Puebla e Hidalgo es superior a los 19,4 kg/m $\mathrm{m}^{-2}$ reportados por Ortega-Martínez et al. (20I4) en Puebla y semejante a los 22,8, 26,3 y 29,I kg/m² reportados por Aguilar-Gallegos, Muñoz-Rodríguez, Santoyo-Cortés y Aguilar-Ávila (2013b), que constituyen medias nacionales. Además, se obtienen rendimientos promedio superiores a los reportados en Almería, de 20,9 kg/m² (Valera Martínez, Belmonte Ureña, Molina Aiz y López Martínez, 2014).

Es relevante mencionar que las innovaciones menos adoptadas por los agricultores, dentro de la categoría Organización, son las relacionadas con el control administrativo y con las ventas y compras en común. En lo que respecta a la categoría Proceso, las innovaciones menos adoptadas son las relacionadas con el manejo y monitoreo del balance nutrimental y el manejo de agroquímicos, lo que acelera la salinización del suelo, y con respecto a los agroquímicos, estos incrementan el riesgo de intoxicación de los trabajadores y la generación de resistencia en las plagas y enfermedades.

En la categoría de Producto, las prácticas menos adoptadas se relacionan con el uso de productos orgánicos; esta situación limita el acceso a mercados con precios más estables y atractivos. Por último, en la categoría de Mercado, la exploración de nuevos mercados es prácticamente inexistente, lo que aumenta la dependencia de los intermediarios. Lo anterior podría deberse a que, tradicionalmente, el enfoque en el desarrollo agrícola ha sido la innovación tecnológica (nuevas variedades o razas, tipos de equipos o métodos de control de plagas). Sin embargo, ahora se reconoce cada vez más que las innovaciones sociales e institucionales pueden ser tan importantes como las técnicas.

\section{Nivel tecnológico}

De acuerdo con la clasificación sobre el nivel tecnológico de los invernaderos propuesta por Costa y Giacomelli (2005), es importante mencionar que en las tres regiones estudiadas se encontró que las primeras construcciones de invernaderos tenían diseños heterogéneos, de herrería, cristal, fibra de vidrio y paredes de ladrillo, que por lo general eran las propuestas tecnológicas para la producción de otros cultivos, como, por ejemplo, la producción de flor de corte.

En México, el nivel tecnológico presenta variaciones que se relacionan con las condiciones geográficas y climáticas de cada región. Sin embargo, de acuerdo con Kuss et al. (2016), por ejemplo, Sinaloa, que es uno de los estados donde se concentran las mayores superficies de agricultura protegida, se enfoca más en la producción con tecnología de casa sombra, mientras que la región centro adopta mayor equipamiento debido a las condiciones de clima más frío. Con respecto a España (Valera Martínez et al., 20I4), se tiene mayor equipamiento, pero menor en comparación con Holanda (Hoffman y Loeber, 2015). Sin embargo, solo un bajo porcentaje de la producción logra ingresar de forma exitosa al mercado internacional.

Lo anterior sugiere que la tecnología evolucionó para adaptarse a las condiciones climáticas de las regiones y a la demanda de los mercados, lo que propició una intensa interacción entre productores, constructores, universidades y el Gobierno y tuvo como resultado la institucionalización de la norma mexicana NMX-E-255CNCP-2008 (Centro de Normalización y Certificación de Productos, 2008), un instrumento que ayudó a uniformizar la calidad de las construcciones. 


\section{Sistemas de innovación}

A pesar de que se cuenta con una buena cantidad y diversidad de centros de investigación y universidades configurando los sistemas de innovación, su interacción directa con los agricultores es limitada. Esta situación es aprovechada por los proveedores de insumos y servicios, que no siempre son la mejor opción y en ocasiones generan efectos negativos. En ese sentido, de acuerdo con Bullinger, Auernhammer y Gomeringer (2004), las universidades y los centros de investigación tienen un impacto valioso sobre el proceso de creación de conocimiento e innovación y contribuyen a reducir estos efectos negativos.

Uno de los efectos negativos más importantes está orientado a la compra de soluciones tecnológicas de alto costo por parte de los agricultores. Si bien los proveedores de insumos y servicios son una fuente importante de información, de nuevas tecnologías e innovaciones (Sligo, Massey y Lewis, 2005; Solano et al., 2003; Thuo et al., 20I4; Wood et al., 20I4), su rol está orientado a la venta de soluciones, que no son necesariamente las que los agricultores necesitan (García-Sánchez et al., 20II). Así mismo, se crea y aumenta una dependencia de empresas transnacionales para el mejoramiento genético, la nutrición vegetal y el control de plagas y enfermedades. Además, se incrementan las externalidades negativas de la agricultura protegida, debido a que no se consideran de manera correcta los efectos ambientales del uso intensivo de agroquímicos.

Otro aspecto que es importante considerar es el fomento de una mayor interacción entre los distintos actores. Además, como lo mencionan Pound y Conroy (2017), la innovación social entre los productores puede ser formal o informal e incluye el desarrollo de cooperativas, grupos de agricultores y grupos de autoayuda. De acuerdo con lo anterior, sería conveniente propiciar nuevos tipos de colaboración entre productores o el desarrollo de nuevas redes entre productores, instituciones, clientes y proveedores de servicios que contribuyan a reducir los costos de transacción y a generar economías de escala.

Los resultados sugieren que es necesario desarrollar articuladores sistémicos que se encarguen de mejorar la interacción entre todos los actores de los sistemas (Klerkx, Hall y Leeuwis, 2009). En el sector agrícola, se ha encontrado que la presencia de articuladores es determinante en la difusión y adopción de innovaciones (Batterink, Wubben, Klerkx y Omta, 20ı0; Klerkx y Aarts, 20I3). Con relación a lo anterior, las acciones promovidas por la Unidad Técnica Especializada en Gestión de la Innovación han desarrollado la función de articuladores sistémicos, generando evidencia empírica sobre la mejor interacción entre los diferentes actores del sistema de innovación y su relación con la mejora de indicadores técnicos, productivos y económicos (Aguilar Ávila, Rendón Medel, Muñoz Rodríguez, Altamirano Cárdenas y Santoyo Cortés, 20II).

\section{Comercialización}

La producción de jitomate en México tiene una gran dependencia del mercado internacional. En ese sentido, cuando se presentan problemas en el comercio internacional, por ejemplo, de disminución de las exportaciones, disminución en los precios internacionales o barreras no arancelarias, la producción de los estados líderes se destina al mercado nacional, lo que presiona el precio nacional a la baja. Es importante considerar esta situación: por ejemplo, de acuerdo con Heijden y Vink (2013), es recomendable que el gobierno implemente una serie de acciones orientadas a la provisión de información de mercados y conocimientos técnicos, además de la búsqueda de oportunidades en redes agroalimentarias alternativas.

Por otra parte, las barreras que tienen los pequeños agricultores para acceder a mejores mercados se relacionan con el poco volumen de producción y con la constancia y la calidad que demandan las tiendas de autoservicio o 
las cadenas de restaurantes, lo que concuerda con lo encontrado por Lugo-Morin (2013) y Michelson, Reardon y Perez (20I2). De acuerdo con Moustier, Tam, Anh, Binh y Loc (2010) y Reardon, Timmer y Minten (20I2), para superar estas barreras es necesario que los agricultores lleven a cabo una serie de acciones colectivas orientadas a alcanzar el volumen y la calidad demandada, así como a mejorar la logística para entregar los productos en la frecuencia y los lugares especificados.

\section{Análisis de rentabilidad}

La agricultura protegida ha perdido rentabilidad a través del tiempo. Por ejemplo, Terrones Cordero y Sánchez Torres (20II), en su investigación sobre rentabilidad de la producción de jitomate en invernadero en la región de Hidalgo, econtraron que la relación B-C obtenida para el 2008 fue de I,5 a 3. Un caso similar ocurre en los resultados obtenidos por Rucoba et al. (2006) cuando analizan la misma actividad en Chihuahua, en la cual obtienen una relación B-C de I,89. La relación B-C calculada en esta investigación fue de I,64 y i,09. Aunado a lo anterior, de acuerdo con Kuss et al. (20I6), es posible que esto se deba a los problemas que este tipo de agricultura protegida presenta, como la deficiente vinculación comercial, el escaso capital de trabajo, la baja capacidad para hacer frente a los fenómenos meteorológicos y el limitado acceso a créditos.

En general, de acuerdo con los resultados obtenidos, las perspectivas para el crecimiento de la agricultura protegida son positivas. Sin embargo, si en el largo plazo se mantienen las actuales condiciones de mercado y no se mejora la utilidad de las unidades de producción, que permita a los agricultores sostener un nivel de vida acorde con sus necesidades y expectativas, se conducirá a realizar actividades complementarias o a abandonar la actividad.

Desde el punto de vista económico, la agricultura protegida es más rentable en comparación con los sistemas de producción agrícola tradicional predominante, como la producción de maíz. En promedio, esta actividad no presenta utilidades o son muy bajas (Ayala-Garay, Schwentesius-Rindermann, Preciado-Rangel, Almaguer-Vargas y Rivas-Valencia, 2013; Centro Internacional de Mejoramiento de Maíz y Trigo [CIMMyT], 2017). Otra de las ventajas que ofrece la agricultura protegida es su capacidad de generación de empleo, menos remunerado en el medio rural, así como la incentivación a la creación de nuevas empresas, lo que favorece la creación de economía de aglomeración y potencializa su capacidad de detonar el desarrollo regional.

\section{Conclusiones}

Los sistemas de innovación se encuentran en estado germinal, y si bien los actores aportan distintos recursos (económicos, de conocimiento y tecnológicos, etcétera) para consolidar la agricultura protegida como una estrategia de desarrollo rural, esto no se logra debido a que sus esfuerzos se diluyen por la baja articulación del sistema. Además, los actores focalizan sus recursos en el subsidio de activos y, en menor grado, en la generación y difusión de conocimiento e información.

La poca articulación del sistema y de su orientación da como consecuencia una baja adopción de innovaciones de organización, mercado y producto. Esta situación impide beneficiarse de las ventajas relacionadas con las economías de escala y con la búsqueda y el aprovechamiento de nuevos nichos de mercado. Además, para disminuir la vulnerabilidad de estos sistemas de producción, relacionada con la incertidumbre del mercado, es necesaria la diversificación y diferenciación de la producción, ya que en la actualidad los sistemas de innovación en las regiones se orientan a un solo producto. 
Las características de los agricultores, la presencia de universidades y centros de investigación y la participación del Estado y otros actores constituyen los elementos básicos para activar los sistemas de innovación que favorecerían la creación y aplicación de conocimiento. Sin embargo, es conveniente propiciar nuevos tipos de colaboración y nuevas redes entre productores, instituciones, clientes y proveedores de insumos y servicios que permitan el desarrollo de un sistema de innovación inclusivo e interactivo como la base para mejorar el desempeño de la agricultura protegida y su impacto en el desarrollo de las regiones. Esto podría lograrse mediante instrumentos de política pública que incentiven la participación de articuladores sistémicos para que coordinen y armonicen los objetivos, las acciones y las decisiones de los actores del sistema de innovación.

Finalmente, el enfoque de sistemas de innovación para analizar los sistemas de producción en los territorios permite identificar sus actores y los recursos que cada uno de estos aportan y analizar las relaciones que establecen; esto facilita identificar las áreas de mejora en los procesos de generación, difusión y adopción de innovaciones. De tal manera, las organizaciones públicas encargadas de diseñar e implementar estrategias para el desarrollo rural deben migrar de una intervención lineal, individual y focalizada en la mejora de los activos productivos a la activación de un sistema de innovación que priorice la generación y aplicación de conocimiento acorde con el nivel de madurez de los sistemas de producción y las condiciones socioterritoriales.

\section{Agradecimientos}

Los autores de este trabajo agradecen a todos los agricultores que colaboraron en este proyecto, por su invaluable apoyo, confianza y esfuerzo para producir alimentos de calidad y contribuir al desarrollo de un campo mexicano competitivo y dar a sus familias una mejor calidad de vida.

\section{Referencias}

Agricultural \& Applied Economics Association [AAEA]. (2000). Commodity costs and returns estimation handbook. Iowa: autor.

Aguilar Ávila, J., Rendón Medel, R., Muñoz Rodríguez, M., Altamirano Cárdenas, J. R., y Santoyo Cortés, V. H. (20II). Agencias para la gestión de la innovación en territorios rurales. En M. del Roble (ed.), Territorio y ambiente: aproximaciones metodológicas (pp. 79-98). Siglo XXI.

Aguilar-Gallegos, N., Muñoz-Rodríguez, M., Santoyo-Cortés, V. H., y Aguilar-Ávila, J. (201za). Influencia del perfil de los productores en la adopción de innovaciones en tres cultivos tropicales. Teuken Bidikay, (4), 207-228. Recuperado de https://www.researchgate.net/publication/264311724_Influencia_del_perfil_de_los_p roductores_en_la_adopcion_de_innovaciones_en_tres_cultivos_tropicales

Aguilar-Gallegos, N., Muñoz-Rodríguez, M., Santoyo-Cortés, V. H., y Aguilar-Ávila, J. (2013b). Políticas públicas para el fomento de clústers de horticultura protegida con pequeños productores: lecciones aprendidas. Reporte de Investigación 93. Texcoco: Centro de Investigaciones Económicas Sociales y Tecnológicas de la Agroindustria y la Agricultura Mundial (Ciestaam). https://doi.org/IO.I3140/2.I.4073.0409

Asociación Mexicana de Horticultura Protegida A. C. [Amhpac]. (2009). Estudio de oportunidades externas para el desarrollo de la inteligencia comercial del mercado de exportación de la horticultura protegida. Reporte final. México: autor.

Ayala-Garay, A. V., Schwentesius-Rindermann, R., Preciado-Rangel, P., Almaguer-Vargas, G., y Rivas-Valencia, P. (2013). Análisis de rentabilidad de la producción de maíz en la región de Tulancingo, Hidalgo, México. Agricultura, Sociedad y Desarrollo, io(4), 38I-395. Recuperado de http://www.scielo.org.mx/scielo.php?scri pt=sci_arttext\&pid=SI870-5472201300040000I 
Aznar, A. (20II). El clúster agroindustrial de la horticultura intensiva de Almería: surgimiento, dinámica y perspectivas. Cuadernos de Estudios Agroalimentarios, 2, 199-217. Recuperado de https://www.researchgate.net/publication/270162433_El_cluster_agroindustrial_de_la_horticultura_intens iva_de_Almeria_surgimiento_dinamica_y_perspectivas

Bastida, T. A. (2008). Los invernaderos en México. Chapingo: Universidad Autónoma Chapingo.

Batterink, M. H., Wubben, E. F. M., Klerkx, L., y Omta, S. W. F. (Onno). (2010). Orchestrating innovation networks: The case of innovation brokers in the agri-food sector. Entrepreneurship \& Regional Development, 22, 47-76. https://doi.org/10.1080/08985620903220512

Borbón Morales, C. G., y Arvizu Armenta, M. (2015). Contraste empírico de la transferencia de tecnología en tres empresas de agricultura protegida en México. Nova Scientia, 7(15), 364-388. Recuperado de http://www.sci elo.org.mx/scielo.php?script=sci_arttext\&pid=S2007-07052015000300364

Bullinger, H. J., Auernhammer, K., y Gomeringer, A. (2004). Managing innovation networks in the knowledge-driven economy. International Journal of Production Research, 42(17), 3337-3353. https:// doi.org/10.1080/00207540410001695970

Caravaca, I., González, G., García, A., Fernández, V., y Mendoza, A. (2014). Conocimiento, innovación y estrategias públicas de desarrollo: análisis comparado de tres ciudades medias de andalucía (España). Revista Latinoamericana de Estudios Urbanos Regionales (EURE), 40(I19). http://doi.org/10.4067/ So250-71612014000100003

Carlsson, B., Jacobsson, S., Holmén, M., y Rickne, A. (2002). Innovation systems: Analytical and methodological issues. Research Policy, 31, 233-245. https://doi.org/10.1016/S0048-7333(0I)00138-X

Castañeda-Miranda, R., Ventura-Ramos, E., Peniche-Vera, R. del, y Herrera-Ruiz, G. (2007). Análisis y simulación del modelo físico de un invernadero bajo condiciones climáticas de la región central de México. Agrociencia, 4I(3), 317-335. Recuperado de http://www.redalyc.org/articulo.oa?id=30241307

Centro Internacional de Mejoramiento de Maíz y Trigo [CIMMyT]. (20I7). Red de plataformas de investigación MasAgro. Resultados PV 2016 y OI 2016-17. En S. Fonteyne y N. Verhulst (eds.). Texcoco: autor.

Centro de Normalización y Certificación de Productos. (2008). Norma Mexicana para el Diseño y Construcción de Invernaderos.

Costa, P., y Giacomelli, G. (2005). Los planes del éxito. Agricultura protegida: productividad basada en el nivel tecnológico. Productores de Hortalizas para Centroamérica (USA), 7(I), 42-44.

Edquist, C. (1997). Systems of innovation: Technologies, institutions, and organizations. Psychology Press.

Edquist, C. (Junio de 200I). The systems of innovation approach and innovation policy: An account of the state of the art. Trabajo presentado en la conferencia Druid, Aalborg.

Elliott, F. F. (1928). The "representative firm” idea applied to research and extension in agricultural economics. Journal of Farm Economics, IO(4), 195-204. Recuperado de http://www.jstor.org/stable/ı229927

Galdeano-Gómez, E., Aznar-Sánchez, J. A., y Pérez-Mesa, J. C. (20II). The complexity of theories on rural development in Europe: An analysis of the paradigmatic case of Almería (South-east Spain). Sociologia Ruralis, 5I(I), 54-78. https://doi.org/I0.IIII/j.I467-9523.2010.00524.X

García López, R., y García Moreno, M. (2010). La gestión para resultados en el desarrollo. América Latina y el Caribe (2.a ed.). Banco Interaméricano de Desarrollo.

García-Sánchez, I. E., Aguilar-Ávila, J., y Bernal-Muñoz, R. (20II). La agricultura protejida en Tlaxcala, México. La adopción de innovaciones y el nivel de equipamiento como factores para su categorización. Teuken Bidikay, 2, 193-212. Recuperado 
de https://www.researchgate.net/publication/274376296_La_agricultura_protegida_en_Tlaxcala_Mejico_la_a dopcion_de_innovaciones_y_el_nivel_de_equipamiento_como_factores_para_su_categorizacion

García Torrente, R., y Perez Mesa, J. C. (20I2). Invernaderos, innovacion para la productividad y el medio ambiente. Cuaderno de Estudios Agroalimnetarios, (I), II5-138. Recuperado de http://www.publicacionescajamar.es/p $\mathrm{df} /$ publicaciones-periodicas/cuadernos-de-estudios-agroalimentarios-cea/3/3-548.pdf

Gittinger, J. P. (1983). Análisis económico de proyectos agrícolas (2.a ed., vol. I). Madrid: Instituto de Desarrollo Económico del Banco Mundial. https://doi.org/10.1017/CBO9781107415324.004

Goodman, L. A. (196I). Snowball Sampling. The Annals of Mathematical Statistics, 32(I), I48-170. Recuperado de https://www.jstor.org/stable/2237615

Hamui-Sutton, A., y Varela-Ruiz, M. (2013). La técnica de grupos focales. Investigación en Educación Médica, 2(I), 55-60. https://doi.org/10.1016/S2007-5057(13)72683-8

Heijden, T. van der, y Vink, N. (2013). Good for whom? Supermarkets and small farmers in south africa-a critical review of current approaches to increasing access to modern markets. Agrekon, 52(I), 68-86. https:// doi.org/10.1080/03031853.2013.778466

Hekkert, M. P., Suurs, R. A. A., Negro, S. O., Kuhlmann, S., y Smits, R. E. H. M. (2007). Functions of innovation systems: A new approach for analysing technological change. Technological Forecasting and Social Change, 74(4), 413-432. https://doi.org/10.1016/j.techfore.2006.03.002

Hoffman, J., y Loeber, A. (2015). Exploring the micro-politics in transitions from a practice perspective: The case of greenhouse innovation in the Netherlands. Journal of Environmental Policy \& Planning, i8(5), 692-7II. https://doi.org/10.1080/1523908x.2015.1113514

Instituto Nacional de Estadística y Geografía [Inegi]. (2007). VII Censo Nacional Agropecuario y Forestal.

Instituto Nacional de Estadística y Geografía [Inegi]. (2009). Prontuario de información geográfica municipal de los Estados Unidos Mexicanos. Jiménez: autor. Recuperado de http://wwwz.inegi.org.mx/contenidos/app /mexicocifras/datos_geograficos/08/08036.pdf

Instituto Nacional de Estadística y Geografía [Inegi], y Secretaría de Agricultura, Ganadería, Desarrollo Rural, Pesca y Alimentación [Sagarpa]. (2015). Encuesta Nacional Agropecuaria (ENA) 20I4. Información Relevante.

Klerkx, L., Hall, A., y Leeuwis, C. (2009). Strengthening agricultural innovation capacity: Are innovation brokers the answer? International Journal of Agricultural Resources, Governance and Ecology, 8(5/6), 409. https:// doi.org/10.1504/IJARGE.2009.032643

Klerkx, L., y Aarts, N. (2013). The interaction of multiple champions in orchestrating innovation networks: Conflicts and complementarities. Technovation, 33(6-7), 193-210. https://doi.org/10.1016/ j.technovation.2013.03.002

Kuss, E., Flores, D., y Harrison, T. (2016). Mexico tomato annual: Mexico continues to expand greenhouse tomato production (Número de reporte: MX602I).

Lane, D., y Maxfield, R. (1996). Strategy under complexity: Fostering generative relationships. Long Range Planning, 29(2), 215-231. https://doi.org/10.1016/0024-630I(96)000II-8

Lugo-Morin, D. R. (2013). Supermercados, estrategias y pequeños productores hortícolas en el municipio de Acatzingo: el caso Walmart. Economía, Sociedad y Territorio, 13(42), 315-349. Recuperado de http://www.sc ielo.org.mx/scielo.php?script=sci_arttext\&pid=SI405-84212013000200003

Lundvall, B. Å. (1992). National systems of innovation: Toward a theory of innovation and interactive learning. Londres: Anthem Press. 
Max, J. F. J., Schurr, U., Tantau, H. J., Mutwiwa, U. N., Hofmann, T., y Ulbrich, A. (20I2). Greenhouse cover technology. Horticultural Reviews, 40(I), 259-396. https://doi.org/I0.1002/978III835187ı.ch7

Michelson, H., Reardon, T., y Perez, F. (2012). Small farmers and big retail: Trade-offs of supplying supermarkets in Nicaragua. World Development, 40(2), 342-354. https://doi.org/10.1016/j.worlddev.2011.07.013

Moreno, R. A., Aguilar, D. J., y Luévano, G. A. (20rI). Características de la agricultura protegida y su entorno en México. Revista Mexicana de Agronegocios, 15(29), 763-774. Recuperado de http://www.redalyc.org/articu lo.oa?id=I41190520I4

Moustier, P., Tam, P. T. G., Anh, D. T., Binh, V. T., y Loc, N. T. T. (2010). The role of farmer organizations in supplying supermarkets with quality food in Vietnam. Food Policy, 35(I), 69-78. https://doi.org/I0.I0I6/ j.foodpol.2009.08.003

Muñoz-Rodríguez, M., Rendón-Medel, R., Aguilar-Ávila, J., García-Muñiz, J. G., y Altamirano-Cárdenas, J. R. (2004). Redes de innovación: un acercamiento a su identificación, análisis y gestión para el desarrollo rural. Chapingo: Universidad Autónoma Chapingo, Fundación Produce Michoacán.

Organización de las Naciones Unidas para la Agricultura y la Alimentación [FAO], y Secretaría de Agricultura, Ganadería, Desarrollo Rural, Pesca y Alimentación [Sagarpa]. (2014). Estudio sobre el envejecimiento de la población rural en México. Ciudad de México: autores.

Organización para la Cooperación y el Desarrollo Económicos [OCDE]. (2004). Science and Innovation Policy. Key challenges and opportunities. París: Development Organisation for Economic Co-operation and Development.

Organización para la Cooperación y el Desarrollo Económicos [OCDE]. (2005). Manual de Oslo. La medida de las actividades científicas y tecnológicas, guía para la recogida e interpretación de datos sobre innovación (3.a ed., vol. 30). Autor. https://doi.org/10.1787/9789264065659-es

Ortega-Martínez, L. D., Ocampo-Mendoza, J., Sandoval-Castro, E., Martínez-Valenzuela, C., Huerta de la Peña, A., y Jaramillo-Villanueva, J. L. (2014). Caracterización y funcionalidad de invernaderos en Chignahuapan, Puebla, México. Bio Ciencias, 2(4), 26I-270. https://doi.org/10.1574I/revbio.02.04.04

Perera, M. S., Sivayoganathan, C., y Wijeratne, M. (2003). Technical knowledge and adoption of farming practices to farmer level extension communication of outgrower farmers of Sri Lankan sugar industry. Sugar Tech, 5(3), I2I-I29. https://doi.org/I0.1007/BFo2943622

Pound, B., y Conroy, C. (2017). The innovation systems approach to agricultural research and development. Agricultural systems: Agroecology and rural innovation for development. Elsevier. https://doi.org/I0.I0I6/ B978-0-I2-802070-8.0001I-6

Ramírez Valverde, B., Ramírez Valverde, G., Juárez Sánchez, J. P., y Cesin Vargas, A. (2007). Tecnología e implementos agrícolas: estudio longitudinal en una región campesina de Puebla, México. Revista de Geografía Agrícola, 38, 55-70. Recuperado de http://www.redalyc.org/articulo.oa?id=75703806

Ramos Gourcy, F. (I4 de mayo del 20I4). Capacitación e integración esenciales para desarrollo y consolidación de la agricultura protegida en México. Hortalizas. Recuperado de https://www.hortalizas.com/horticultura-protegida/capacitacion-e-integracion-esenciales-para-desarroll o-y-consolidacion-de-la-agricultura-protegida-en-mexico/

Reardon, T., Timmer, C. P., y Minten, B. (2012). Supermarket revolution in Asia and emerging development strategies to include small farmers. Proceedings of the National Academy of Sciences, I09(3I), I2332-I2337. https://doi.org/10.1073/pnas.1003160108 
Rucoba, G. A., Anchondo, N. A., Luján, Á. C., y Olivas, G. J. M. (2006). Análisis de rentabilidad de un sistema de producción de tomate bajo invernadero en la región centro-sur de Chihuahua. Revista Mexicana de Agronegocios, IO(19), I-IO. http://www.redalyc.org/articulo.oa?id=I4101909

Ruiz, M., Ruiz, J., Torres, V., y Cach, J. (2012). Estudio de sistemas de producción de carne bovina en un municipio del estado de Hidalgo, México. Revista Cubana de Ciencia Agrícola, 46(3), 26I-265. Recuperado de http:// www.redalyc.org/articulo.oa?id=193025294006

Sagarnaga, M., Ochoa, R. F., Salas, J. M., Anderson, D. P., Richardson, J. W., y Knutson, R. D. (1999). Granjas porcinas representativas en México: panorama económico 1995-2004. Texas.

Sakib, H., e Islam Afrad, S. (20I4). Adoption of modern aquaculture technologies by the fish farmers in Bogra district of Bangladesh. International Journal of Agriculture Innovations and Research, 3(2), 414-42I. Recuperado de https://www.researchgate.net/publication/277535477_Adoption_of_Modern_Aquaculture_T echnologies_by_the_Fish_Farmers_in_Bogra_District_of_Bangladesh

Santos, B. M., Obregón-Olivas, H. A., y Salamé-Donoso, T. P. (2010). Producción de hortalizas en ambientes protegidos: estructuras para la agricultura protegida. Departamento de Horticultural Sciences, Servicio de Extensión Cooperativa de la Florida. Recuperado de https://horticulture.ucdavis.edu/sites/g/files/dgvnsk I816/files/extension_material_files/Santos_academic_paper_estructuras_para_la_agricultura_protegida.pdf

Secretaría de Agricultura, Ganadería, Desarrollo Rural, Pesca y Alimentación [Sagarpa], y Servicio de Información Agroalimentaria y Pesquera [SIAP]. (2013). Datos abiertos; datos de exportaciones e importaciones.

Secretaría de Agricultura, Ganadería, Desarrollo Rural, Pesca y Alimentación [Sagarpa], y Servicio de Información Agroalimentaria y Pesquera [SIAP]. (2015). Superficie cubierta y número de instalaciones de agricultura protegida por el Servicios de Informaión Agrolimentaria y Pesquera (SIAP). Autores.

Sligo, F. X., Massey, C., y Lewis, K. (2005). Informational benefits via knowledge networks among farmers. Journal of Workplace Learning, I7(7), 452-466. https://doi.org/10.1108/13665620510620034

Smith, E. G., Richardson, J. W., Anderson, D. P., Gray, A. W., Klose, S. L., Knutson, R. D., ... Schwart, R. B. (1997). Representative farms economic outlook for the november 1997 FAPRI/AFPC baseline. AFPC Work. 97-13. Recuperado de https://www.researchgate.net/publication/23516008_Representative_Farms_Economic _Outlook_For_The_November_1997_FapriAfpc_Baseline

Solano, C., León, H., Pérez, E., Herrero, M., León, H., Pérez, E., y Herrero, M. (2003). The role of personal information sources on the decision-making process of Costa Rican dairy farmers. Agricultural Systems, 76(I), 3-18. https://doi.org/10.1016/S0308-52IX(02)00074-4

Spielman, D. J., Davis, K., y Negash, M. (20II). Rural innovation systems and networks: Findings from a study of Ethiopian smallholders. Agric Hum Values (201I), 28, 195-212. https://doi.org/10.1007/s10460-010-9273-y

Spielman, D. J., Ekboir, J., Davis, K., y Ochieng, C. M. O. (2008). An innovation systems perspective on strengthening agricultural education and training in sub-Saharan Africa. Agricultural Systems, 98(I), I-9. https://doi.org/10.1016/j.agsy.2008.03.004

Teddlie, C., y Yu, F. (2007). Mixed methods sampling: A typology with examples. Journal of Mixed Methods Research, I(I), 77-I00. https://doi.org/10.1177/2345678906292430

Terrones Cordero, A., y Sánchez Torres, Y. (20II). Análisis de la rentabilidad económica de la producción de jitomate bajo invernadero en Acaxochitlán, Hidalgo. Revista Mexicana de Agronegocios, 15(29), 752-76I. Recuperado de http://www.redalyc.org/articulo.oa?id=I4II90520I3

Thuo, M., Bell, A. A., Bravo-Ureta, B. E., Lachaud, M. A., Okello, D. K., Okoko, E. N., ... Puppala, N. (20I4). Effects of social network factors on information acquisition and adoption of improved groundnut varieties: 
The case of Uganda and Kenya. Agriculture and Human Values, 31(3), 339-353. https://doi.org/10.1007/ sIO460-0I4-9486-6

Valera Martínez, D. L., Belmonte Ureña, L. J., Molina Aiz, F. D., y López Martínez, A. (20I4). Los invernaderos de Almería. Análisis de su tecnología y rentabilidad. España: Cajamar Caja Rural.

Vargas-Canales, J. M., Castillo-González, A. M., Pineda-Pineda, J., Ramírez-Arias, J. A., y Avitia-García, E. (20I4). Nutrient extraction of tomato (Solanum lycopersicum L.) in mixtures of volcanic rock with fresh and recycled sawdust. Revista Chapingo Serie Horticultura, 20(I), 7I-88. https://doi.org/I0.5154/r.rchsh.2013.02.005

Vargas-Canales, J. M., Palacios-Rangel, M. I., Aguilar-Ávila, J., Ocampo-Ledesma, J. G., y Medina-Cuellar, S. E. (20I8). Efficiency of small enterprises of protected agriculture in the adoption of innovations in Mexico. Estudios Gerenciales, 34(I46), 52-62. https://doi.org/10.18046/j.estger.2018.I46.28II

Vargas-Canales, J. M., Palacios-Rangel, M. I., Aguilar-Ávila, J., y Ocampo-Ledesma, J. G. (2016). Cambio tecnológico e innovación en agricultura protegida en Hidalgo, México. Chapingo: Universidad Autónoma Chapingo. Recuperado de http://ciestaam.edu.mx/cambio-tecnologico-e-innovacion-en-agricultura-protegi da-en-hidalgo-mexico/

Vargas-Canales, J. M., Palacios-Rangel, M. I., Camacho-Vera, J. H., Aguilar-Ávila, J., y Ocampo-Ledesma, J. G. (2015). Factores de innovación en agricultura protegida en la región de Tulancingo, México. Revista Mexicana de Ciencias Agri\#colas, 6(4), 827-840. Recuperado de http://www.scielo.org.mx/scielo.php?script=sci_arttex t\&pid=S2007-09342015000400013

Vázquez-Barquero, A., y Rodríguez-Cohard, J. C. (2016). Endogenous development and institutions: Challenges for local development initiatives. Environment and Planning C: Government and Policy, 34(6), II35-1153. https://doi.org/10.1177/0263774XI5624924

Wood, B. A., Blair, H. T., Gray, D. I., Kemp, P. D., Kenyon, P. R., Morris, S. T., y Sewell, A. M. (2014). Agricultural science in the wild: A social network analysis of farmer knowledge exchange. PLoS ONE, 9(8). https:// doi.org/10.1371/journal.pone.0105203

\section{Notas}

*Artículo de investigación

Licencia Creative Commons CC-BY 4.0 